\title{
Behçet's disease with multiple splenic abscesses in a child
}

\author{
Kyung In Lim, Dong Hwa Yang, Eell Ryoo \\ Department of Pediatrics, Gachon University Gil Hospital, Incheon, Korea
}

We report the case of a 5-year-old male patient with multiple aseptic splenic abscesses associated with Behçet's disease. The patient visited Gachon University Gil Hospital with fever, abdominal pain, and acute watery and bloody diarrhea, and reported a 2-year history of chronic abdominal pain and intermittent watery diarrhea. He was treated with antibiotics at a local clinic for fever and cervical lymph node swelling. Additionally, he had recurrent stomatitis. A colonoscopy showed multiple well-demarcated ulcerations throughout the colon, and abdominal computed tomography showed multiple splenic abscesses. Pathergy and HLA-B51 tests were positive. Investigations did not reveal any infectious organisms in the aspirate obtained via ultrasoundguided fine needle aspiration. After steroid treatment, all symptoms and multiple aseptic splenic abscesses resolved. However, oral ulcers, genital ulcers, and abdominal pain recurred after tapering the steroids. Infliximab treatment improved the patient's symptoms. However, 5 months after the treatment, the symptoms recurred. The treatment was changed to include adalimumab. Subsequently, the patient's symptoms resolved and colonoscopic findings improved. No recurrence was noted after 3 months of follow-up. (Intest Res 2017;15:422-428)

Key Words: Behcet syndrome; Spleen; Abscess; Pediatrics

\section{INTRODUCTION}

Behçet's disease (BD) is a systemic inflammatory disorder characterized by recurrent mouth ulcers, general symptoms such as genital ulceration, ocular, dermatologic, gastrointestinal, neurological, and vascular disease, and arthritis. ${ }^{1}$ When BD is accompanied by gastrointestinal symptoms such as abdominal pain and diarrhea with typical ulcerative lesions, it can be diagnosed as intestinal $\mathrm{BD}{ }^{2}$ While $\mathrm{BD}$ is mostly diagnosed in adults in their twenties and thirties, ${ }^{1}$ intestinal BD is usually diagnosed in adults in their thirties. ${ }^{3}$ The prevalence of $\mathrm{BD}$ is higher in Asia and the Middle East than in Western countries, and $3 \%$ to $16 \%$ of $\mathrm{BD}$ patients also

Received May 31, 2016. Revised August 31, 2016.

Accepted September 12, 2016. Published online February 21, 2017 Correspondence to Eell Ryoo, Division of Pediatric Gastroenterology, Hepatology and Nutrition, Department of Pediatrics, Gachon University Gi Hospital, 21 Namdong-daero 774 beon-gil, Namdong-gu, Incheon 21565, Korea. Tel: +82-32-460-3224, Fax:+82-32-460-2362, E-mail: ryoo518@ gilhospital.com

Financial support: None. Conflict of interest: None. have intestinal involvement. ${ }^{1}$ Few large-scale studies have investigated childhood BD due to the small number of cases, although patients with BD may have a family history, indicating a genetic predisposition. ${ }^{4,5}$ Although a study by Turkish researchers found no significant difference in gastrointestinal involvement between adults and children, ${ }^{4}$ a study by Japanese researchers indicated that gastrointestinal involvement occurred more frequently in children. ${ }^{6}$

Aseptic abscesses (AAs) can be found in many diseases. The condition is accompanied by fever, weight loss, and abdominal pain, and is found to improve with corticosteroids but not with antibiotics. Neutrophilic infiltration is found in biopsies. ${ }^{7}$ In systemic diseases in both children and adults, AAs have been reported, mostly in association with IBD and sometimes with Sweet's syndrome or pyoderma gangrenosum. ${ }^{7-11}$ AAs associated with BD are rare in adults ${ }^{12}$ and has not been reported in children.

We present a case of a 5-year-old boy with BD accompanied by multiple splenic AAs that was managed with pharmaceutical therapy.

\footnotetext{
๑ Copyright 2017. Korean Association for the Study of Intestinal Diseases. All rights reserved.

This is an Open Access article distributed under the terms of the Creative Commons Attribution Non-Commercial License (http://creativecommons.org/licenses/by-nc/4.0)

which permits unrestricted non-commercial use, distribution, and reproduction in any medium, provided the original work is properly cited.
} 


\section{CASE REPORT}

A 5-year-old boy visited the hospital with a 6-day history of fever and abdominal pain, and a 2-year history of intermittent abdominal pain and diarrhea. The abdominal pain occurred 3 to 4 times per week. Watery diarrhea occurred 5 times a day with 3 to 4 episodes of mucoid bloody diarrhea since 3 days ago. Cervical lymph node enlargement developed 10 days before the hospital visit. Antibiotics were administered at a primary care clinic for 6 days, but failed to alleviate the fever. The patient exhibited failure to thrive. His height was $100 \mathrm{~cm}$ (third to fifth percentile) and weight was $14 \mathrm{~kg}$ (<third percentile). He also experienced recurrent stomatitis.

A physical examination showed bilateral cervical lymph node enlargement without tenderness. Bilateral pharyngeal injection and pharyngotonsillar hypertrophy were observed $(2+)$. A whitish exudate was observed without oral ulcers. Bowel sounds were increased. Periumbilical, epigastric, and left lower quadrant tenderness was found, but there was no rebound tenderness. No abnormal skin lesions were found.

Blood tests revealed the following: hemoglobin $10.5 \mathrm{~g} / \mathrm{dL}$, hematocrit 33.3\%, white blood cells 30,500/mm (80.1\% neutrophils), platelets 586,000/mm, CRP $21.63 \mathrm{mg} / \mathrm{dL}$, AST 17
U/L, ALT $6 \mathrm{U} / \mathrm{L}$, total bilirubin $0.3 \mathrm{mg} / \mathrm{dL}, \gamma$-glutamyl transferase $12 \mathrm{U} / \mathrm{L}$, ALP $153 \mathrm{U} / \mathrm{L}$, amylase $37 \mathrm{U} / \mathrm{L}$, and lipase 15 $\mathrm{U} / \mathrm{L}$. Additionally, stool was positive for occult blood.

Antibiotics were administered for tonsillitis and cervical lymphadenitis, but the patient's fever did not subside. Further examinations were conducted to identify the source of the fever. Repeated blood tests showed anemia with hemoglobin $8.0 \mathrm{~g} / \mathrm{dL}$ and hematocrit $25.8 \%$. Leukocytosis was observed with white blood cells $25,620 / \mathrm{mm}$, although all infectionrelated tests were negative. Tuberculosis was ruled out with negative results for interferon- $\gamma$ release assays (QuantiFER$\mathrm{ON}^{\circledR}$ ) and sputum analysis. Autoantibody tests were negative for ANA, rheumatoid factor, antineutrophil cytoplasmic antibodies, and anti-Saccharomyces cerevisiae antibodies. No hematologic malignancy was observed on peripheral blood smear. Abdominal CT revealed no definite abnormalities in the small or large intestine. However, lesions suggestive of multiple abscesses were revealed in the spleen (Fig. 1B), the largest one being $2.3 \times 1.6 \times 1.9 \mathrm{~cm}$. Abdominal ultrasonography revealed multiple splenic abscesses, the largest being $0.88 \mathrm{~cm}$ (Fig. 2A). The pediatric infection department recommended that we perform ultrasound-guided fine needle aspiration to determine whether the abscesses were septic and start a 2-week course of conventional antibiotics for the
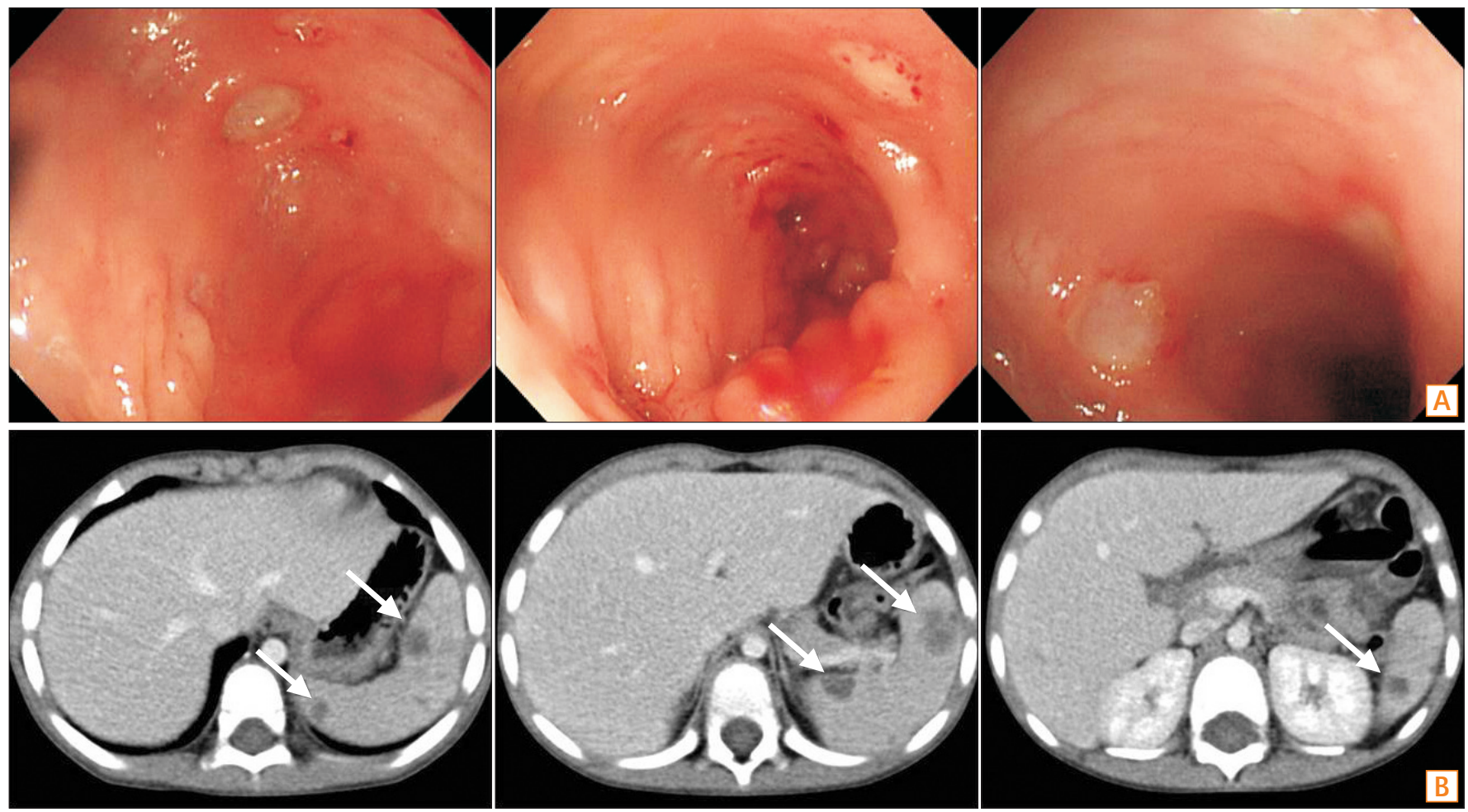

Fig. 1. Colonoscopic and abdominal CT findings at initial visit. (A) Multiple well demarcated ulcerations that covered with whitish plaques were noted on the cecum, A-colon, T-colon and D-colon. And friability was also noted near the ulcerative lesion. (B) Multiple abscesses were noted on the spleen (arrows). 

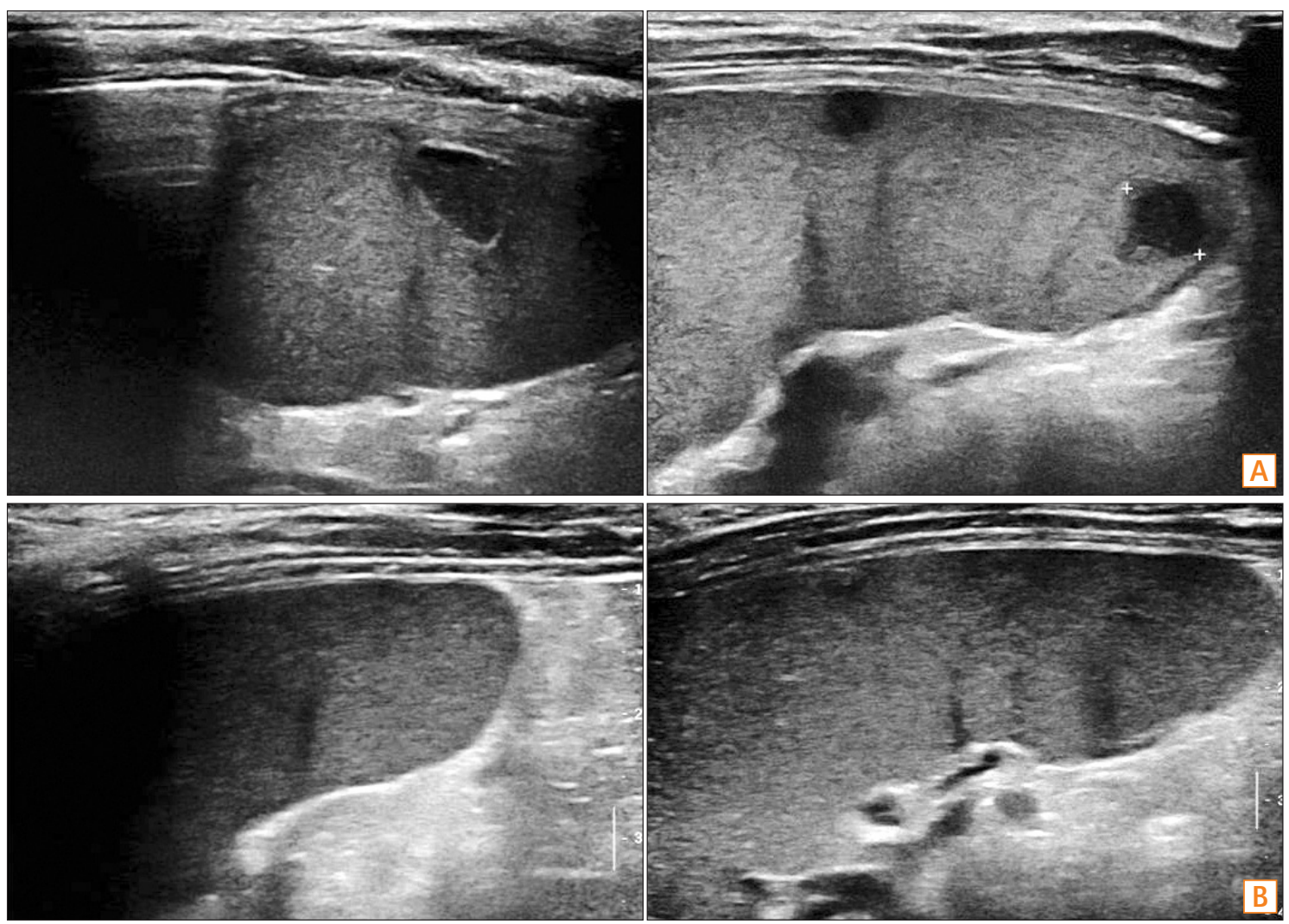

Fig. 2. Abdominal ultrasonographic findings. (A) Before steroid therapy, multiple splenic abscesses were observed. The largest one is $0.88 \mathrm{~cm}$ sized. (B) After steroid therapy, multiple splenic abscesses were subsided at 1 month later.

splenic abscesses. Therefore, fine needle aspiration of one splenic abscess was performed, and $4 \mathrm{~mL}$ of bloody and puslike fluid was aspirated. After the 2-week course of antibiotic treatment, follow-up abdominal CT still showed splenic abscesses, and fever was persistent. A PCR test for tuberculosis from the aspirated fluid of multiple AAs was negative and no bacteria and fungus were cultured in the AAs.

A colonoscopy performed due to the persistent fever and continuous bloody stools uncovered multiple circular or oval ulcers throughout the colon with a thick white coating covering the base, indicating BD (Fig. 1A). Erosion and nonspecific inflammation were found, but no granulomas were observed on pathology (Fig. 3). Pathergy and HLA-B51 gene tests for BD were positive. An ophthalmologic examination identified no pathology.

The patient had recurrent mouth ulcers, a positive pathergy test, and positive findings on colonoscopy. He was diagnosed with incomplete BD (probable intestinal BD) with multiple AAs in the spleen. Prednisone $1 \mathrm{mg} / \mathrm{kg} / \mathrm{day}$ and 5 -aminosalicylic acid $100 \mathrm{mg} / \mathrm{kg} /$ day were given for 1 month. After treatment, improvements in clinical symptoms and blood tests were noted. An abdominal ultrasound showed that the splenic abscesses had disappeared (Fig. 2B). Treatment side effects including a moon face were observed, and the steroids were tapered. The patient visited the hospital 1 month later for worsening mouth ulcers, diarrhea, and abdominal pain. He received prednisone $1 \mathrm{mg} / \mathrm{kg} /$ day again with the addition of azathioprine $1 \mathrm{mg} / \mathrm{kg} /$ day. His condition improved 1 month later, and the drug dosage was reduced.

However, his condition worsened 1 week later, and he was again emergently hospitalized. By the time he was admitted, he complained of mouth ulcers, abdominal pain, proctodynia, and penile pain. He was diagnosed with complete BD (definite intestinal BD) after multiple mouth, anal, and penile tip ulcers (Fig. 4A) along with general abdominal tenderness were found on physical examination. The disease activity index for intestinal Behçet's disease (DAIBD) score was 195. Splenic abscesses were not found on abdominal CT. Multiple ulcers were found at different locations on colonoscopy, when compared with the previous exam (Fig. 4B).

It was determined that the intestinal $\mathrm{BD}$ was re-aggravated and an infliximab $5 \mathrm{mg} / \mathrm{kg}$ dose was administered. Improvement was observed in both clinical aspects and physical examination, and the DAIBD score decreased to 30. After the 

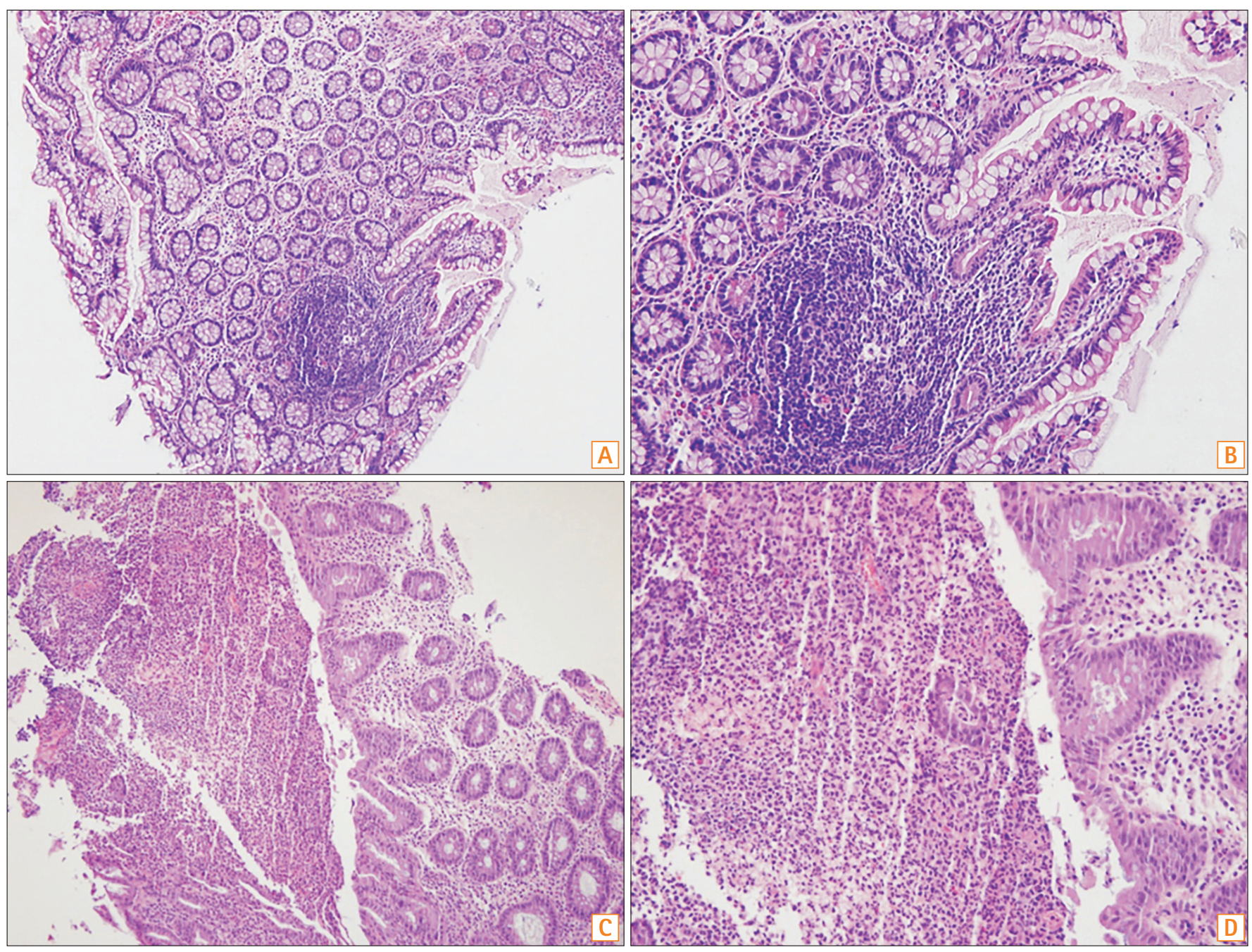

Fig. 3. Pathologic findings. Erosion and nonspecific inflammation were found but no granuloma was observed in ileum and total colon. (A) Ileum $(\mathrm{HEE}, \times 100)$, (B) ileum $\left(H \& \mathrm{H}_{1} \times 200\right),(\mathrm{C})$ colon $(\mathrm{HEtE}, \times 100)$, and (D) colon $(\mathrm{HEE}, \times 200)$.

initial infliximab dose, the second $5 \mathrm{mg} / \mathrm{kg}$ dose was administered 2 weeks later, and the third dose 4 weeks after that. Subsequently, the patient received one $5 \mathrm{mg} / \mathrm{kg}$ dose every 8 weeks.

Five months after the first administration of infliximab, the patient was hospitalized for recurrent abdominal pain, fever, and oral ulcers. The DAIBD score increased to 125 . After other causes of acute abdomen such as acute appendicitis were ruled out, treatment comprising colchicine $(0.5 \mathrm{mg} / \mathrm{kg} / \mathrm{day})$ for the oral ulcer, prednisone ( $1 \mathrm{mg} / \mathrm{kg} /$ day), azathioprine ( $1 \mathrm{mg} / \mathrm{kg} /$ day), and $80 \mathrm{mg}$ of adalimumab for re-aggravated intestinal BD were administered. The patient's symptoms resolved after the adalimumab treatment, the DAIBD score decreased to 15, and he was discharged from the hospital. The steroid was tapered, and $40 \mathrm{mg}$ of adalimumab was administered after the first injection, followed by $20 \mathrm{mg}$ of adalimumab every 2 weeks after the second injection. A follow-up colonoscopy performed 3 months postdischarge found old scars of previous ulcerations in the terminal ileum, cecum, and colon (Fig. 5).

Since the first administration of adalimumab, the patient regularly followed up every 2 weeks for adalimumab injections, observation of symptoms, and physical examination. Recurrence was not observed during the 3-month follow-up period.

\section{DISCUSSION}

BD is diagnosed based on clinical signs rather than specific tests. The diagnostic criteria proposed by the International Study Group in 1999 remain widely used till date. According to those criteria, a patient may be diagnosed with $\mathrm{BD}$ when 

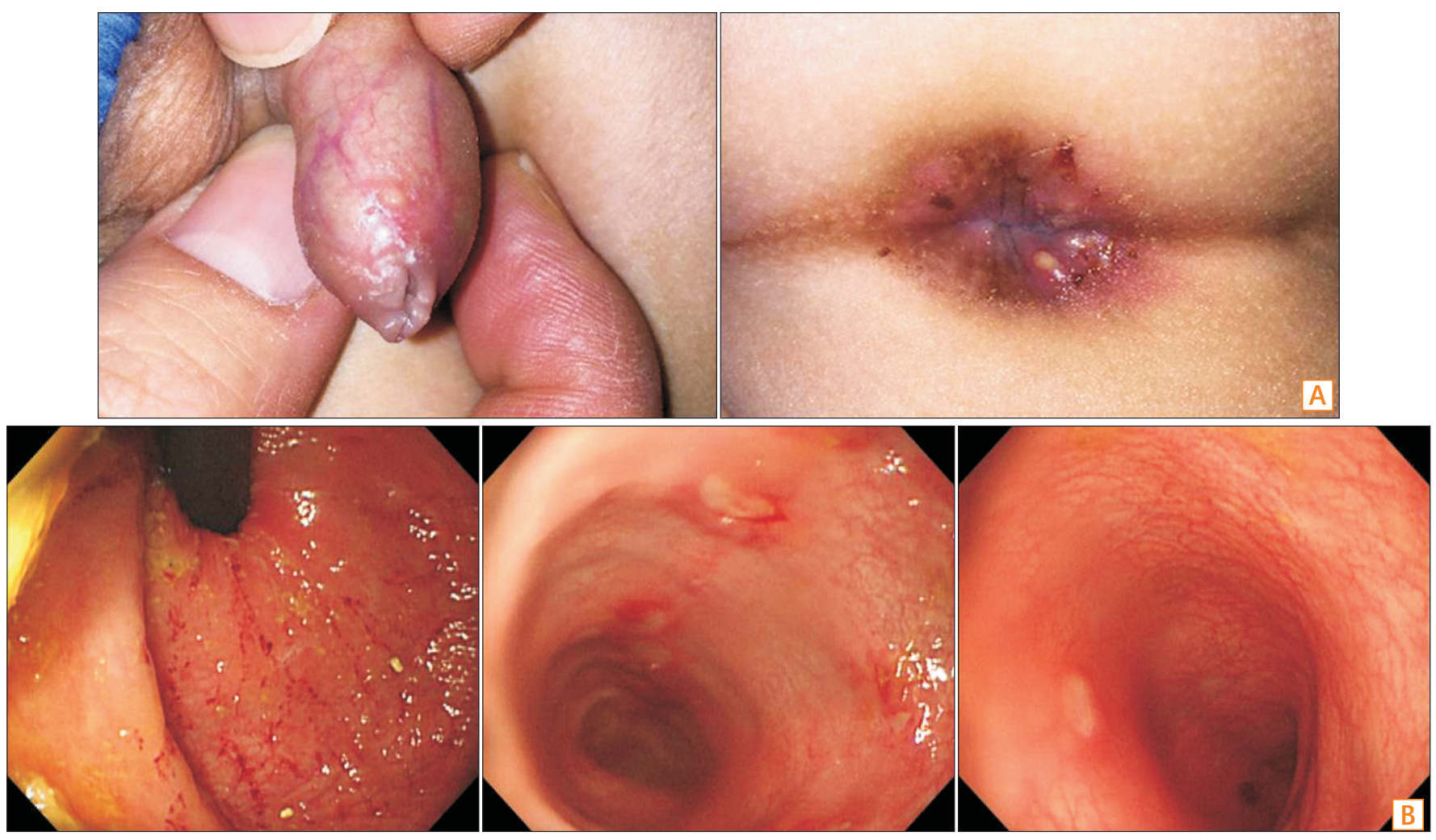

Fig. 4. Re-aggravation of genital, perianal and intestinal Behçet's disease after steroid tapering. (A) Ulcerations were observed on the penis tip and perianal area. (B) Multiple round well demarcated ulcerations were noted on the T- and D-colon. And hemorrhagic erosive lesions were noted on the rectum.
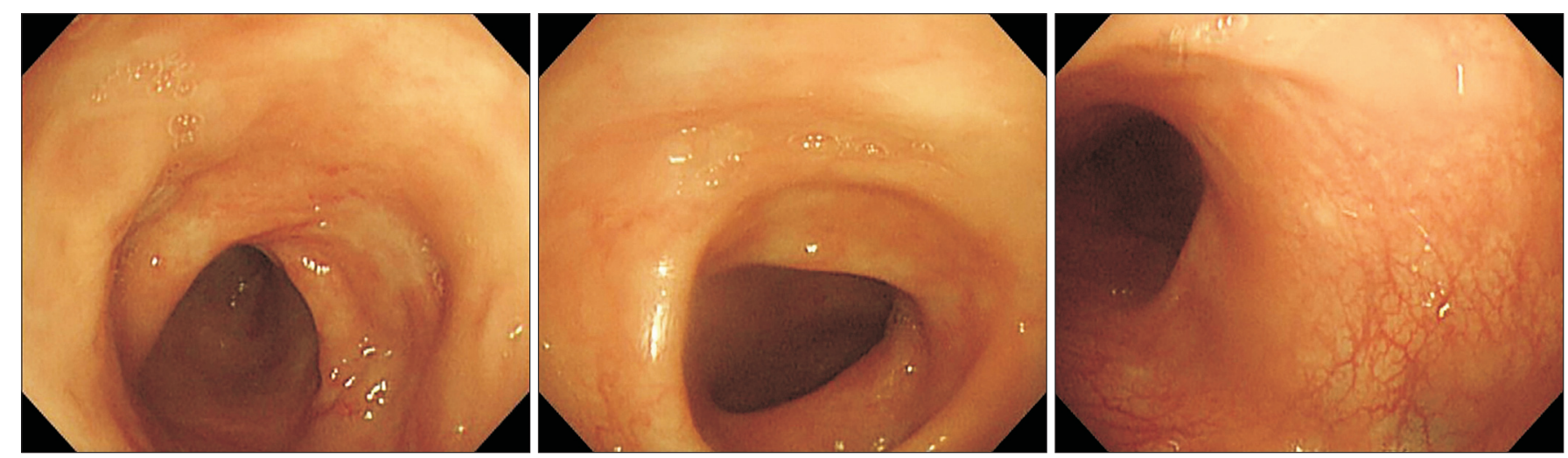

Fig. 5. Follow-up colonoscopic findings after adalimumab administration. Some old scars of previous ulceration were found in terminal ileum, cecum, and colon.

two of the following criteria are met: (1) recurrent genital ulcers; (2) eyeball lesions, such as anterior or posterior uveitis and retinal vasculitis; (3) skin lesions, such as pseudofolliculitis and erythema nodosum; and (4) a positive pathergy test. ${ }^{13}$

Cheon et al. ${ }^{14}$ conducted a study limiting the diagnostic algorithm for intestinal BD to intestinal canal ulcers and clinical aspects, and classified them into types, i.e., definite, probable, and suspected, based on the shape observed on endoscopy and whether there were general symptoms. However, in-depth tracking observation was required, as patients who did not initially meet the diagnostic criteria for BD frequently developed general symptoms later., ${ }^{2,14,15}$ The patient in our study had recurrent mouth ulcers and was pathergy-positive but did not meet International Study Group criteria. However, BD-related ulcer lesions were identified with endoscopy and he tested positive for HLA-B51. 
Therefore, his condition was considered incomplete BD with probable intestinal BD. Since general symptoms alone were insufficient to confirm the diagnosis, CD was also considered. However, since the endoscopic and pathologic findings were strongly suggestive of BD and general symptoms of BD including genital and oral ulcers appeared later, he was eventually diagnosed with definite intestinal BD.

For intestinal BD, empirical therapies have been applied in individual cases and retrospective trials, but no standardized treatments have been determined using a case-controlled study. In 2007, Kobayashi et al. ${ }^{16}$ suggested treatment standards based on disease severity, including 5 -aminosalicylic acid, steroids, immunosuppressive drugs, enteral nutrition, total parenteral nutrition, and surgical resection as well as experimental treatments such as infliximab, colchicine, leukocytapheresis, thalidomide, antibiotics, and endoscopic ethanol spray. Similar treatments were reported for childhood intestinal BD, and symptoms reportedly resolved with infliximab for steroid-resistant (refractory) BD. ${ }^{17,18}$

Intestinal $\mathrm{BD}$, like $\mathrm{CD}$, can present with repeated cycles of recurrence, requiring treatment. For this reason, disease activity should be monitored routinely. Cheon et al. ${ }^{19}$ developed the DAIBD in 2007 and classified intestinal BD as minor, moderate, and major based on the presence of general symptoms for a week, fever, number of extraintestinal manifestations, abdominal pain severity, palpable abdominal masses, abdominal tenderness, number of gastrointestinal complications, and number of diarrheal episodes.

The DAIBD was completed for this case when the condition recurred, and steroid/immuno-suppressive agent-resistant (refractory) BD was diagnosed. Infliximab was administered and the disease severity improved from moderate to minor.

The following criteria are used to define AA: (1) deep abscess(es) on radiologic examination with neutrophilic features proven by surgical pathology or aspiration; (2) negative blood cultures, negative serologic tests for bacteria including Yersinia enterocolitica and, when a surgical procedure or aspiration was performed, sterile standard AFB and fungal cultures of pus are observed (studies were left to the clinician's discretion); (3) antibiotic therapy failure, when prescribed, after at least 2 weeks' duration for conventional antibiotic therapy and 3 months' duration for antituberculosis drugs that were continued for a conventional duration; and (4) rapid clinical improvement the day after corticosteroid initiation (at least $0.5 \mathrm{mg} / \mathrm{kg}$ of prednisone or equivalent; $1 \mathrm{mg} /$ $\mathrm{kg}$ recommended) followed by radiologic improvement after 1 month of corticosteroids, which is occasionally adminis- tered in combination with immunosuppressive drugs. ${ }^{7}$ Since the clinical manifestation and laboratory findings in this case met AA criteria, the multiple splenic abscesses were defined as AAs.

Generally, AAs are usually found with IBD, and there are reports about the association between AAs and uncommon inflammatory diseases such as Sweet's syndrome and pyoderma gangrenosum, both of which are characterized by specific skin lesions. This patient did not have any skin lesions, which ruled these disorders out.

AAs is rarely found in adults, and few cases have been reported in children. Among adults, AAs accompanied by $\mathrm{BD}$ are also rarely reported. A case was recently reported in which multiple abscesses were observed in the liver and spleen. ${ }^{12}$ The steroid dosage was increased when the condition recurred after initial steroid tapering, and the AAs eventually improved. However, there have been no reports of AAs associated with BD in children. Treatment in this case was based on the therapeutic protocol for adult BD with AAs. All of the AAs improved after the administration of steroids for 1 month, and the symptoms of intestinal BD improved as well. Although the intestinal BD recurred and worsened during the steroid tapering, the AAs did not recur. This is the first known case report of AAs in childhood BD.

\section{REFERENCES}

1. Sakane T, Takeno M, Suzuki N, Inaba G. Behçet's disease. N Engl J Med 1999;341:1284-1291.

2. Hisamatsu T, Naganuma M, Matsuoka K, Kanai T. Diagnosis and management of intestinal Behçet's disease. Clin J Gastroenterol 2014;7:205-212.

3. Yang SK. Intestinal Behcet's disease. Intest Res 2005;3:1-10.

4. Karincaoglu Y, Borlu M, Toker SC, et al. Demographic and clinical properties of juvenile-onset Behçet's disease: a controlled multicenter study. J Am Acad Dermatol 2008;58:579-584.

5. Al Mosawi ZS, Madan W, Fareed E. Pediatric-onset Behçet disease in Bahrain: report of nine cases and literature review. Arch Iran Med 2012;15:485-487.

6. Tabata M, Tomomasa T, Kaneko H, Morikawa A. Intestinal Behçet's disease: a case report and review of Japanese reports in children. J Pediatr Gastroenterol Nutr 1999;29:477-481.

7. André MF, Piette JC, Kémény JL, et al. Aseptic abscesses: a study of 30 patients with or without inflammatory bowel disease and review of the literature. Medicine (Baltimore) 2007;86:145-161.

8. Quilichini R, Mazzerbo F, Baume D, Carsuzaa F, Burtey S. Sweet's syndrome and aseptic abscess of the spleen. Rev Med Interne 1996;17:1029-1031. 
9. Klinger S, Mathis N, Jackson S. Bullous Sweet syndrome associated with an aseptic splenic abscess. Cutis 2009;84:255-258.

10. Fukuhara K, Urano Y, Kimura S, Hori K, Arase S. Pyoderma gangrenosum with rheumatoid arthritis and pulmonary aseptic abscess responding to treatment with dapsone. Br J Dermatol 1998;139:556-558.

11. Johnson JL, West DA, Haggstrom AN. Pyoderma gangrenosum associated with an aseptic splenic abscess in a patient with neurofibromatosis. Pediatr Dermatol 2015;32:113-117.

12. Maeshima K, Ishii K, Inoue M, Himeno K, Seike M. Behçet's disease complicated by multiple aseptic abscesses of the liver and spleen. World J Gastroenterol 2013;19:3165-3168.

13. International Study Group for Behçet's Disease. Criteria for diagnosis of Behçet's disease. Lancet 1990;335:1078-1080.

14. Cheon JH, Kim ES, Shin SJ, et al. Development and validation of novel diagnostic criteria for intestinal Behçet's disease in Korean patients with ileocolonic ulcers. Am J Gastroenterol 2009;104:2492-2499.
15. Cheon JH, Shin SJ, Kim SW, et al. Diagnosis of intestinal Behçet's disease. Korean J Gastroenterol 2009;53:187-193.

16. Kobayashi K, Ueno F, Bito S, et al. Development of consensus statements for the diagnosis and management of intestinal Behçet's disease using a modified Delphi approach. J Gastroenterol 2007;42:737-745.

17. Kinoshita H, Kunisaki R, Yamamoto H, et al. Efficacy of infliximab in patients with intestinal Behçet's disease refractory to conventional medication. Intern Med 2013;52:1855-1862.

18. Caporuscio S, Pranteda G, Nistico S, et al. An incomplete form of childhood Behçet's disease treated with infliximab. Int J Immunopathol Pharmacol 2014;27:445-448.

19. Cheon JH, Han DS, Park JY, et al. Development, validation, and responsiveness of a novel disease activity index for intestinal Behçet's disease. Inflamm Bowel Dis 2011;17:605-613. 